\title{
Structural and Magnetic Properties of Dilute Spinel Ferrites: Neutron Diffractometry and Magnetometry Investigations
}

\author{
H. Mamiya ${ }^{1 *}$, N. Terada ${ }^{1}$, H. Kitazawa ${ }^{1}$, A. Hoshikawa ${ }^{2}$, and T. Ishigaki ${ }^{2}$ \\ ${ }^{1}$ National Institute for Materials Science, Tsukuba 305-0047, Japan \\ ${ }^{2}$ Ibaraki University, Mito 310-8512, Japan
}

(Received 25 January 2011, Received in final form 16 February 2011, Accepted 12 April 2011)

\begin{abstract}
Magnetic properties of highly zinc-substituted manganese ferrites are discussed on the basis of cation distribution. High throughput neutron powder diffractometry indicates that the prepared samples possess a nearly normal spinel structure, where the substitution of nonmagnetic zinc ions mainly causes the dilution of magnetic ions in the A-sublattice and consequently affects bond-randomness in the B-sublattice. On the other hand, the estimated occupancy of manganese ions in the B site indicates that random anisotropy effects due to local Jahn-Teller distortions gradually weaken with the substitution. Bulk magnetometry indicates that the substitution smears the transition from a paramagnetic phase to a soft-magnetic phase. Furthermore, at lower temperatures, such a soft-magnetic phase is destabilized and a magnetic glassy state appears. These features of the magnetic properties of dilute spinel ferrites are discussed from the viewpoint of the above-mentioned various types of disorders.
\end{abstract}

Keywords : oxide spinel, TOF neutron diffraction, diluted ferrimagnet, smeared phase transition, reentrant spin-glass

\section{Introduction}

One reason for the renewed interest in photomagnetic [1] and magnetocaloric [2] effects in highly zinc-substituted manganese ferrites in recent times is that ubiquitous magnets such as ferrites have advantages over newly discovered magnetic materials. For example, to employ magnetic refrigeration in mass-produced appliances such as air conditioners and refrigerators, magnetic refrigerants must be made from easily produced metals such as $\mathrm{Mg}$, $\mathrm{Al}, \mathrm{Mn}, \mathrm{Fe}, \mathrm{Zn}, \mathrm{Cd}$, and $\mathrm{Pb}$; however, because household utensils must be nontoxic, $\mathrm{Cd}$ and $\mathrm{Pb}$ are excluded. Furthermore, these magnetic materials require durability against oxidation and high heat cycles. For these reasons, we take a fresh look at the usefulness of ferrites [3] for such new types of applications.

Manganese zinc ferrites, $\mathrm{Mn}_{1-\mathrm{x}} \mathrm{Zn}_{\mathrm{x}} \mathrm{Fe}_{2} \mathrm{O}_{4}$, possess basic $\mathrm{AB}_{2} \mathrm{O}_{4}$ spinel structure in which oxygen has fcc close packing (O:32e), and cations can occupy either tetrahedral (A:8a) or octahedral (B:16d) interstitial sites [4]. Hence, the structure of spinel ferrite is divided into two extremes by the arrangement of cations in the A and B sites: normal-

*Corresponding author: Tel: +81-29-859-2755

Fax: +81-29-859-2801, e-mail: MAMIYA.Hiroaki@nims.go.jp spinel (M) $[\mathrm{Fe}]_{2} \mathrm{O}_{4}$ and inverse-spinel $(\mathrm{Fe})[\mathrm{MFe}] \mathrm{O}_{4}$ arrangements, where $\mathrm{M}$ refers to either zinc or manganese ions, and the parentheses and brackets refer to ions occupying the $\mathrm{A}$ and $\mathrm{B}$ sites, respectively. We know that $\mathrm{Zn}^{2+}$ ions strongly prefer the A site, as exemplified by normal spinels $(\mathrm{Zn})[\mathrm{Mn}]_{2} \mathrm{O}_{4}$ and $(\mathrm{Zn})[\mathrm{Fe}]_{2} \mathrm{O}_{4}$ [4]. On the other hand, the actual inversion between manganese and iron ions depends on the synthesis conditions [4-7]. It is difficult, however, to determine the site occupancy of these ions by conventional X-ray diffractometry because their X-ray scattering factors are similar. For this reason, some researchers have recently proposed a two-wave length anomalous dispersion (TWAD) method [5,6] by using synchrotron radiation. On the other hand, the typical measuring time required to obtain "Rietveld-quality" data is substantially long in conventional neutron diffractometry although the neutron scattering length of a manganese nucleus, $b(\mathrm{Mn})$ $=-3.750 \mathrm{fm}$, is significantly different from that of an iron nucleus, $b(\mathrm{Fe})=9.45 \mathrm{fm}$. Therefore, the cation distribution determined by previous structural studies has been frequently referred to in the discussion on the magnetic properties of manganese zinc ferrites, although cation distribution depends on synthesis conditions in each study.

In addition to the difficulty of structural studies, there is room for further investigation on the effects of the sub- 
stitution of zinc ions on magnetic properties. In other words, substitution not only lowers the ordering temperature but also smears the phase transition associated with inhomogeneous short-range ordering [2]. Furthermore, the frustration due to competitive ferromagnetic and antiferromagnetic interactions, which is induced by the substitution, may destroy the long-range order before it reaches the percolation threshold $[2,8,9]$. Such short-range ordering and magnetic frustrations are correlated with the arrangement of zinc, manganese, and iron ions in the A and $\mathrm{B}$ sites. In other words, we must consider different types of disorders such as (1) site-randomness of magnetic ions in the diluted sublattice, (2) bond-randomness in the B-sublattice caused by the random disappearance of the effectively ferromagnetic $\mathrm{B}-\mathrm{A}-\mathrm{B}$ couplings, in addition to constant anti-ferromagnetic B-B couplings $J_{\mathrm{BB}}$, and (3) random anisotropy due to local Jahn-Teller distortions around $\mathrm{Mn}^{3+}$ ions in the octahedral B site [10]. Therefore, careful discussion on the effects of zinc substitution on the basis of knowledge on cation distribution is desired. In this study, we determine the arrangement of cations in highly zinc-substituted manganese ferrites by using a high throughput neutron powder diffractometer, and discuss the relationship between the structural and magnetic properties of the same sample.

\section{Experimental}

Sintered samples of $\mathrm{Mn}_{1-x} \mathrm{Zn}_{x} \mathrm{Fe}_{2} \mathrm{O}_{4}(x=0.60,0.65,0.70$, 0.75 , and 0.80 ) were prepared by a standard solid-state reaction [2]. Appropriate ratios of $\mathrm{ZnO}(99.9 \%), \mathrm{Mn}_{2} \mathrm{O}_{3}$ (99.9\%), and $\mathrm{Fe}_{2} \mathrm{O}_{3}(99.99 \%)$ were mixed and then pressed into pellets. The pellets were annealed in air at $1523 \mathrm{~K}$ for $24 \mathrm{~h}$, and finally quenched in liquid nitrogen.

Neutron powder diffraction patterns were taken using a high throughput neutron powder diffractometer, iMATERIA, installed at the megawatt-class pulsed-spallation neutron sources, J-SNS, at the Japanese particle accelerator research complex (J-PARC). The exposure time for data collection in the $0.12-\mathrm{MW}$ operation was approximately $900 \mathrm{~s}$ for each sample contained in a $6-\mathrm{mm}$ diameter cylindrical vanadium cell. A more detailed description of the diffractometer can be found elsewhere [11]. Rietveld refinements were performed using the program Z-Rietveld ver. 0.9.31 for time-of-flight neutron powder diffraction analyses [12].

Zero-field-cooled magnetization $M_{\mathrm{ZFC}}$ and field-cooled magnetization $M_{\mathrm{FC}}$ were measured by commercial Quantum Design MPMS and PPMS magnetometers. The sweep rate of temperature was $1.0 \mathrm{~K} / \mathrm{min}$. Although the used samples were not ideal ellipsoids, approximate demagnetizing factors, $N_{\mathrm{d}}$, were estimated to be $0.045,0.084,0.034$,
0.012 , and 0.035 for samples with $x=0.60,0.65,0.70$, 0.75 , and 0.80 , respectively. In these cases, observable magnetization $M$ can be considered to be saturated at $H /$ $N_{\mathrm{d}}$ even when the samples are soft-magnetic at lower $H$.

\section{Cation Distribution}

Neutron powder diffraction patterns at room temperature indicate that the samples were made of a single spinel phase, as shown in Fig. 1. No super-lattice peaks were observed. Hence we are certain that zinc ions were randomly substituted for manganese ions. The total diffraction patterns with 384 peaks in the range 35.3-247 pm were used in the Rietveld refinement. All diffraction patterns were refined with the space group $\mathrm{Fd} \overline{3} \mathrm{~m}$ (no. 227, origin choice 1) [13]. The site occupancy of oxygen was fixed at 1.0. On the other hand, the A site can be occupied by zinc, manganese, and iron ions. Therefore, we assume a virtual ion with mean scattering length of the ions in the A site, $<b_{\mathrm{A}}>=\Sigma g_{\mathrm{A}}\left(\mathrm{M}_{\mathrm{j}}\right) \cdot b\left(\mathrm{M}_{\mathrm{j}}\right)$, where $g_{\mathrm{A}}\left(\mathrm{M}_{\mathrm{j}}\right)$ is the occupancy of the $j$-th cation $\mathrm{M}_{\mathrm{j}}$ in the A site, $b\left(\mathrm{M}_{\mathrm{j}}\right)$ is the scattering length of $\mathrm{M}_{\mathrm{j}}$, and the summations are made for the three cations, $\mathrm{M}_{\mathrm{j}}=\mathrm{Zn}, \mathrm{Mn}$, and Fe. Similarly, we consider $\left\langle b_{\mathrm{B}}\right\rangle=\Sigma g_{\mathrm{B}}\left(\mathrm{M}_{\mathrm{j}}\right) \cdot b\left(\mathrm{M}_{\mathrm{j}}\right)$ in the B site. Note that $\left.<b_{\mathrm{A}}\right\rangle$ is given by $[x \cdot b(\mathrm{Zn})+(1-x) \cdot b(\mathrm{Mn})]+\delta b$, while $<b_{\mathrm{B}}>$ is expressed as $b(\mathrm{Fe})-\delta b$ on the constraints $g_{\mathrm{A}}(\mathrm{Zn})$ $+2 g_{\mathrm{B}}(\mathrm{Zn})=x, g_{\mathrm{A}}(\mathrm{Mn})+2 g_{\mathrm{B}}(\mathrm{Mn})=1-x, \Sigma g_{\mathrm{A}}\left(\mathrm{M}_{\mathrm{j}}\right)=1$, and $\Sigma g_{\mathrm{B}}\left(\mathrm{M}_{\mathrm{j}}\right)=1$, where $\delta b=(b(\mathrm{Fe})-b(\mathrm{Zn})) \cdot g_{\mathrm{B}}(\mathrm{Zn})+$ $(b(\mathrm{Fe})-b(\mathrm{Mn})) \cdot g_{\mathrm{B}}(\mathrm{Mn})$. The magnetic scattering is disregarded because the ordering temperature $T_{\mathrm{c}}$ is lower



Fig. 1. Neutron powder diffraction patterns for $\mathrm{Mn}_{1-x} \mathrm{Zn}_{x} \mathrm{Fe}_{2} \mathrm{O}_{4}$ at room temperature. The solid bars show the position of Bragg reflections of a cubic spinel structure. 
Table 1. Refined structural parameters of $\mathrm{Mn}_{1-x} \mathrm{Zn}_{x} \mathrm{Fe}_{2} \mathrm{O}_{4}: x=0.65$ at room temperature, where $U_{\text {iso }}\left(\mathrm{pm}^{2}\right)$ is the isotropic displacement parameter. We also refined the mean scattering length of the ions in the A site, $<b_{\mathrm{A}}>=\sum g_{\mathrm{A}}\left(\mathrm{M}_{\mathrm{j}}\right) \cdot b\left(\mathrm{M}_{\mathrm{j}}\right)$, where $g_{\mathrm{A}}\left(\mathrm{M}_{\mathrm{j}}\right)$ is the occupancy of the $j$-th cation $\mathrm{M}_{\mathrm{j}}$ in the A site, $b\left(\mathrm{M}_{\mathrm{j}}\right)$ is the scattering length of $\mathrm{M}_{\mathrm{j}}$, and the summations are made for the three cations, $\mathrm{M}_{\mathrm{j}}=\mathrm{Zn}, \mathrm{Mn}$, and Fe. Similarly, we refined $<b_{\mathrm{B}}>=\sum g_{\mathrm{B}}\left(\mathrm{M}_{\mathrm{j}}\right) \cdot b\left(\mathrm{M}_{\mathrm{j}}\right)$ in the B site.

\begin{tabular}{ccccccc}
\hline \hline Ion & Site & $\sum g_{\mathrm{k}}\left(\mathrm{M}_{\mathrm{j}}\right) \cdot b\left(\mathrm{M}_{\mathrm{j}}\right)(\mathrm{fm})$ & $x$ & $y$ & $z$ & $U_{\text {iso }}\left(\mathrm{pm}^{2}\right)$ \\
\hline $\mathrm{Zn}, \mathrm{Mn}, \mathrm{Fe}$ & $8 \mathrm{a}$ & $2.94(1)$ & 0 & 0 & 0 & $48(1)$ \\
$\mathrm{Zn}, \mathrm{Mn}, \mathrm{Fe}$ & $16 \mathrm{~d}$ & $9.21(1)$ & 0.625 & 0.625 & 0.625 & $46.7(3)$ \\
$\mathrm{O}$ & $32 \mathrm{e}$ & 5.805 & $0.38580(1)$ & $0.38580(1)$ & $0.38580(1)$ & $58.1(4)$ \\
\hline
\end{tabular}

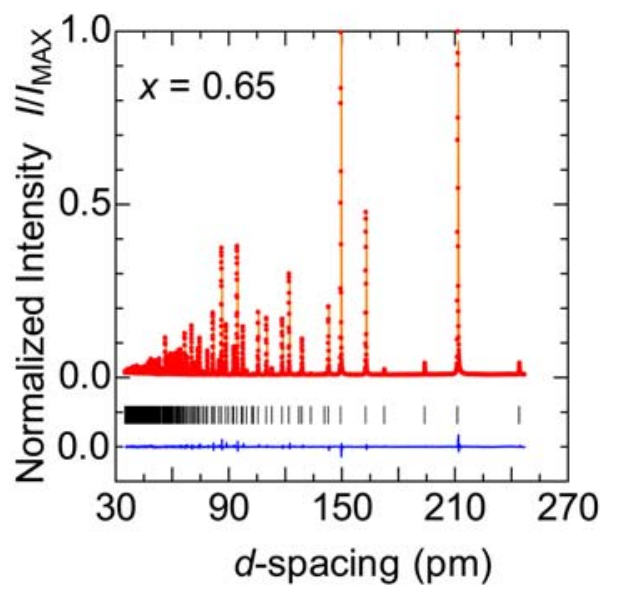

Fig. 2. Observed (circles) and calculated (solid curve) intensities from Rietveld refinements for $\mathrm{Mn}_{1-x} \mathrm{Zn}_{x} \mathrm{Fe}_{2} \mathrm{O}_{4}(x=0.65)$. The difference curve and the position of Bragg reflections are shown in the lower part of the diffractogram.

than the measuring temperature, as will be discussed later. Table 1 shows an example of the refined structural parameters for $\mathrm{Mn}_{1-\mathrm{x}} \mathrm{Zn}_{\mathrm{x}} \mathrm{Fe}_{2} \mathrm{O}_{4}(x=0.65)$. The corresponding fitted and difference curves, and the position of the Bragg reflections are shown in the diffractogram in Fig. 2. Refined parameters at different zinc contents $x$ are shown in Table 2 and also in Fig. 3.

A linear dependence of the lattice constant $a$ on zinc content $x$ can be observed, which is consistent with Vegard's rule. On the other hand, the estimated $\left\langle b_{\mathrm{A}}\right\rangle$ is slightly larger than $[x \cdot b(\mathrm{Zn})+(1-x) \cdot b(\mathrm{Mn})]$, while the

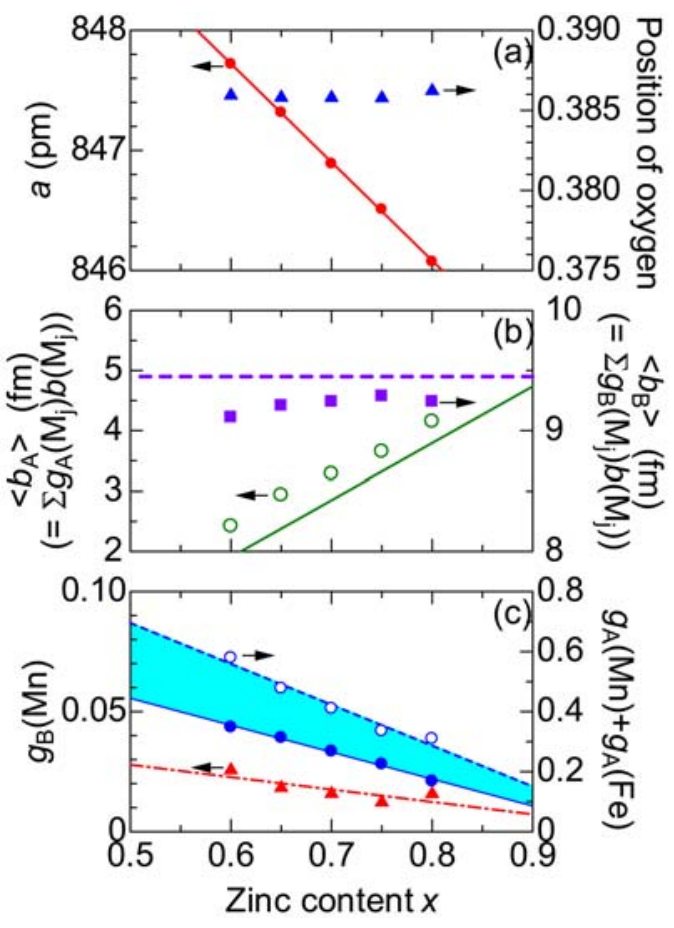

Fig. 3. Zinc content $x$ dependence of the refined parameters: lattice constant, $a$, position of oxygen, mean scattering length of ions in the A sites, $\left\langle b_{\mathrm{A}}\right\rangle=\sum g_{\mathrm{A}}\left(\mathrm{M}_{\mathrm{j}}\right) \cdot b\left(\mathrm{M}_{\mathrm{j}}\right)$ and in the B sites, $<b_{\mathrm{B}}>=\Sigma g_{\mathrm{B}}\left(\mathrm{M}_{\mathrm{j}}\right) \cdot b\left(\mathrm{M}_{\mathrm{j}}\right)$, where $\mathrm{M}_{\mathrm{j}}=\mathrm{Zn}$, Mn, or Fe. The solid line in (b) shows the ideal values given by $[x \cdot b(\mathrm{Zn})+(1-$ $x) \cdot b(\mathrm{Mn})]$, while the broken line in (b) indicates the magnitude of $b(\mathrm{Fe})$. The triangles in (c) show the occupancy of manganese ions in the B sites, $g_{\mathrm{B}}(\mathrm{Mn})$. The solid and open circles in (c) show the total fractions of magnetic ions in the A site in both extreme cases of $g_{\mathrm{B}}(\mathrm{Zn})=0$ and $g_{\mathrm{B}}(\mathrm{Mn})=0$, respectively.

Table 2. R-values, "goodness of fit," gof $=\mathrm{w}_{\mathrm{p}} / R_{\mathrm{e}}$, unit cell parameter, $a(\mathrm{pm})$, mean scattering length of ions in each site, $<b_{\mathrm{A}}>=$ $\sum g_{\mathrm{A}}\left(\mathrm{M}_{\mathrm{j}}\right) \cdot b\left(\mathrm{M}_{\mathrm{j}}\right)$ and $<b_{\mathrm{B}}>=\sum g_{\mathrm{B}}\left(\mathrm{M}_{\mathrm{j}}\right) \cdot b\left(\mathrm{M}_{\mathrm{j}}\right)(\mathrm{fm})$, position of oxygen, Ox, and isotropic displacement parameters, $U_{\text {iso }}\left(\mathrm{pm}^{2}\right)$, from the refinements of the neutron diffraction patterns.

\begin{tabular}{ccccccccccc}
\hline \hline$x$ & $R_{\mathrm{p}}$ & $\mathrm{w} R_{\mathrm{p}}$ & gof & $a(\mathrm{pm})$ & $\begin{array}{c}<b_{\mathrm{A}}>(\mathrm{fm}) \\
: \mathrm{A}(8 \mathrm{a}) \mathrm{site}\end{array}$ & $\begin{array}{c}<b_{\mathrm{B}}>(\mathrm{fm}) \\
: \mathrm{B}(16 \mathrm{~d}) \mathrm{site}\end{array}$ & $\begin{array}{c}\mathrm{Ox} \\
=\mathrm{Oy}=\mathrm{Oz}\end{array}$ & $\begin{array}{c}U_{\text {iso }}\left(\mathrm{pm}^{2}\right) \\
: \mathrm{A}(8 \mathrm{a}) \text { site }\end{array}$ & $\begin{array}{c}U_{\text {iso }}\left(\mathrm{pm}^{2}\right) \\
: \mathrm{B}(16 \mathrm{~d}) \text { site }\end{array}$ & $\begin{array}{c}U_{\text {iso }}\left(\mathrm{pm}^{2}\right) \\
: \mathrm{O}(32 \mathrm{e}) \text { site }\end{array}$ \\
\hline 0.60 & 3.84 & 4.60 & 1.56 & $847.720(1)$ & $2.42(1)$ & $9.11(1)$ & $0.38594(1)$ & $43(1)$ & $47.5(4)$ & $60.8(5)$ \\
0.65 & 3.59 & 4.28 & 1.56 & $847.317(1)$ & $2.94(1)$ & $9.21(1)$ & $0.38580(1)$ & $48(1)$ & $46.7(3)$ & $58.1(4)$ \\
0.70 & 3.69 & 4.59 & 1.64 & $846.890(1)$ & $3.29(1)$ & $9.24(1)$ & $0.38579(1)$ & $48(1)$ & $44.5(3)$ & $56.3(4)$ \\
0.75 & 3.68 & 4.44 & 1.57 & $846.510(1)$ & $3.66(1)$ & $9.29(1)$ & $0.38577(1)$ & $46(1)$ & $42.9(3)$ & $51.9(4)$ \\
0.80 & 3.63 & 4.42 & 1.61 & $846.074(1)$ & $4.16(1)$ & $9.24(1)$ & $0.38622(1)$ & $49(1)$ & $47.6(3)$ & $57.7(4)$ \\
\hline
\end{tabular}


estimated $<b_{\mathrm{B}}>$ is slightly smaller than $b(\mathrm{Fe})$. Because the excess of $\left\langle b_{\mathrm{A}}\right\rangle$ and shortage of $\left\langle b_{\mathrm{B}}>\right.$ are attributed to the same $\delta b$, the site-inversions $g_{\mathrm{B}}(\mathrm{Zn})$ and $g_{\mathrm{B}}(\mathrm{Mn})$ cannot be independently determined by using the refined parameters. Therefore, we assume that $g_{\mathrm{B}}(\mathrm{Zn})=0$ at this stage, because $\mathrm{Zn}^{2+}$ ions strongly prefer the A site, as stated earlier. The triangles in Fig. 3(c) indicate $g_{\mathrm{B}}(\mathrm{Mn})$ estimated from $\left\langle b_{\mathrm{B}}\right\rangle$. In brief, the extent of $g(\mathrm{Mn})$ is a few percent and seems gradually decrease with $x$. Similar results are also obtained on the basis of the excess of $\left\langle b_{\mathrm{A}}\right\rangle$. In other words, the samples prepared with various zinc contents appear to possess a nearly normal spinel structure. Indeed, the positions of oxygen ions estimated at $0.385-0.386$ coincide with the values generally given for a normal spinel [5]. In this context, the random anisotropy effects due to local Jahn-Teller distortions are considered to be gradually weakened with the substitution, because the refinement indicates a decrease in the number of $\mathrm{Mn}^{3+}$ ions surrounded by isotropic $\mathrm{Fe}^{3+}\left(3 \mathrm{~d}^{5}\right)$ ions in the $\mathrm{B}$ site.

We summarize the estimated cation distribution and its influence on magnetic properties as follows: (1) the substitution of nonmagnetic zinc ions mainly causes the dilution of magnetic ions in the A site. Consequently, stochastic inhomogeneity due to the site-randomness in the A-sublattice leads to bases of "Griffiths islands," [14]. (2) On the other hand, spins on the B-sublattice are affected by bond-randomness due to the random disappearance of the effectively ferromagnetic $\mathrm{B}-\mathrm{A}-\mathrm{B}$ couplings. The average of the random bonds varies from ferromagnetic to antiferromagnetic with the substitution, because the other antiferromagnetic couplings $J_{\mathrm{BB}}$ are almost constant in the steady B-sublattice. (3) Random anisotropy effects due to local Jahn-Teller distortions are considered to be gradually weakened with the substitution because the refinement indicates a decrease in the number of $\mathrm{Mn}^{3+}$ ions in the $\mathrm{B}$ site.

Before turning to magnetic properties, we focus on the possibility of $g_{\mathrm{B}}(\mathrm{Zn}) \neq 0$ because nonequilibrium processing, including mechanical milling, coprecipitation, rapid quenching, sputtering, and pulsed-laser deposition, induces the meta-stable phase and disorders the arrangement of $\mathrm{Zn}^{2+}$ and $\mathrm{Fe}^{3+}$ ions $[15,16]$. In a strict sense, we cannot be certain that $g_{\mathrm{B}}(\mathrm{Zn})=0$ even in the bulk specimens synthesized by a solid-state reaction at $1523 \mathrm{~K}$. In our analysis, the magnitude of $\delta b$ indicates that $\mathrm{Zn}^{2+}$ in the $\mathrm{B}$ site is less than $10 \%$ even in the extreme case of $g_{\mathrm{B}}(\mathrm{Mn})=0$. At present, we must recall that even small amounts of nonmagnetic substitution in the B-sublattice seriously affect the magnetic properties when homogeneous antiferromagnetic couplings predominate magnetic interactions between the topologically frustrated spins in the B-sub- lattice $[15,17]$. Such mechanism, however, cannot be directly applied to this system because the effectively ferromagnetic $\mathrm{B}-\mathrm{A}-\mathrm{B}$ couplings are much larger than $J_{\mathrm{BB}}$. For the bond-randomness in the B-sublattice, the disappearance of the $\mathrm{B}-\mathrm{A}-\mathrm{B}$ couplings by decreasing the number of magnetic ions in the A site is, therefore, more important than the slight imperfection of $J_{\mathrm{BB}}$ due to $g_{\mathrm{B}}(\mathrm{Zn}) \neq 0$.

Next, we discuss the role of $\mathrm{Fe}^{3+}$ ions in the A site exchanged for $\mathrm{Zn}^{2+}$ ions. Because $\mathrm{Fe}^{3+}\left(3 \mathrm{~d}^{5}\right)$ ions are equivalent to the isotropic $\mathrm{Mn}^{2+}\left(3 \mathrm{~d}^{5}\right)$ ions, only the total fraction of such magnetic ions in the A site is essential. The solid and open circles in Fig. 3(c) indicate the fractions estimated in both extreme cases of $g_{\mathrm{B}}(\mathrm{Zn})=0$ and $g_{\mathrm{B}}(\mathrm{Mn})=0$, respectively. From the figure, it can be said that the dilution of magnetic ions in the A-sublattice proceeds with the substitution $x$ despite the possibility of $g_{\mathrm{B}}(\mathrm{Zn}) \neq 0$. The above summary would be appropriate even if a small amount of $\mathrm{Zn}^{2+}$ ions existed in the B site, because their influence on magnetic properties seem insignificant.

\section{Magnetic Properties}

Fig. 4 shows temperature dependences of magnetization $M$ for samples with various zinc content $x$. For the sample with low $x(x=0.60)$ we observe different properties in each temperature range: In the highest range of approximately $400 \mathrm{~K}$, the sample is less magnetized even at maximum applied field $H=800 \mathrm{kA} / \mathrm{m}$. In other words, the system is paramagnetic. When the temperature decreases toward room temperature, $M$ increases noticeably. The increase in $M$ at low $H$ is especially rapid. This variation can therefore be attributed to the so-called ferrimagnetic transition. In this temperature range, the fielddependence of $M$ is remarkable in the utilized $H$ range from $4 \mathrm{kA} / \mathrm{m}$ to $800 \mathrm{kA} / \mathrm{m}$. This behavior can be explained by the fact that ordered clusters with various sizes are critically generated in the vicinity of the ferrimagnetic transition temperature $T_{\mathrm{c}}$. Below $T_{\mathrm{c}}, M$ in $H$ lower than 16 $\mathrm{kA} / \mathrm{m}$ flattens out at the extent of $H / N_{\mathrm{d}}$ shown as the broken line. The huge susceptibility indicated by this fact shows that this phase is soft-magnetic. On the other hand, $M$ in higher fields, $H \geq 64 \mathrm{kA} / \mathrm{m}$, linearly increases with decreasing temperature. Because the field-dependence of such $M$ is small in the temperature range $50 \mathrm{~K}$ to $300 \mathrm{~K}$, the sample can be considered to be saturated when the domain structure is annihilated in $H \geq 64 \mathrm{kA} / \mathrm{m}$. The observed linear dependence of saturation magnetization is consistent with that of an R-type ferrimagnet [18]. The local spin arrangement is, however, not a strict collinear 


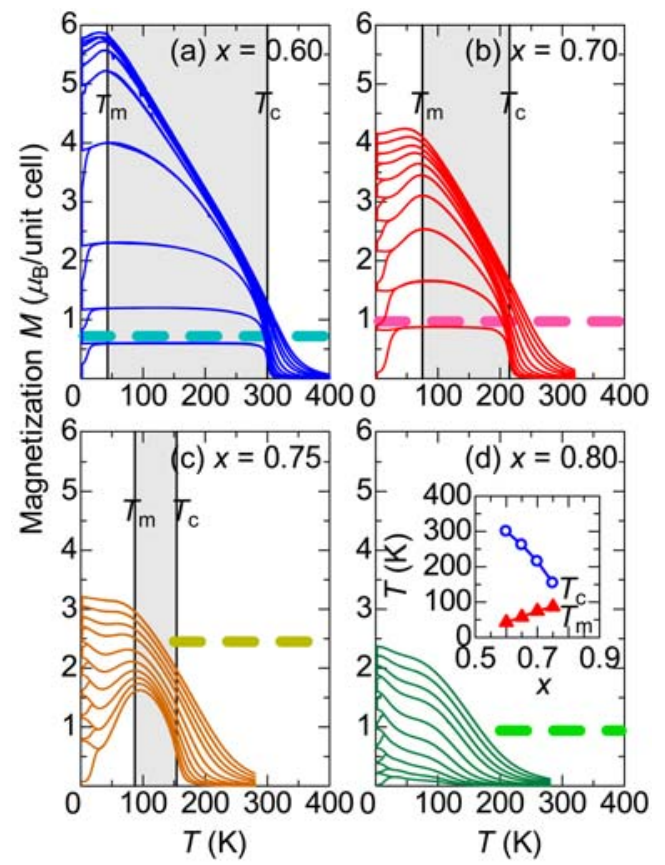

Fig. 4. Temperature dependences of magnetization $M$ for samples with various zinc content $x$, where the upper branch of each curve shows field-cooled magnetization, while the lower branch shows zero-field-cooled magnetization. The applied field $H$ is $4,8,16,32,64,128,256,384,576$, and 800 $\mathrm{kA} / \mathrm{m}$ from bottom to top. The shaded areas denote the intermediate soft-magnetic phase, while the broken lines indicate the extent corresponding to $H / N_{\mathrm{d}}$ at $H=4 \mathrm{kA} / \mathrm{m}$. The inset shows the zinc content dependence of the transition temperature $T_{\mathrm{c}}$ for the transition to the soft-magnetic phase, and of the reentrant transition temperature $T_{\mathrm{m}}$ for the transition to the magnetic glassy phase; the former is estimated from an inflection point of $M_{\mathrm{FC}}(T)$ at $H=4 \mathrm{kA} / \mathrm{m}$ and the latter is the maximum temperature of $M_{\mathrm{FC}}(T)$ at $H=16 \mathrm{kA} / \mathrm{m}$.

order but a locally canted one, because the magnitude of the saturation magnetization is apparently smaller than 8 $\mu_{\mathrm{B}}$ that is expected for the robust collinear order. Note that the locally canted state is considerably stable for the application of $H<800 \mathrm{kA} / \mathrm{m}$. At very low temperatures below a certain temperature $T_{\mathrm{m}} \sim 40 \mathrm{~K}, M_{\mathrm{FC}}$ as well as $M_{\mathrm{ZFC}}$ decreases with decreasing temperature. Because the observed decrease of $M_{\mathrm{FC}}$ cannot be attributed to the pinning of the domain walls, we must consider that the anti-parallel spin arrangement between the A-B sublattices is further destabilized at lower temperatures. In this temperature range, $M_{\mathrm{ZFC}}$ branches off from $M_{\mathrm{FC}}$. When the present results are considered together with supplementary results in a previous paper [9], it can be said that the magnetic state transits to a magnetic glassy phase at low temperatures.

These fundamental properties, first transition to a soft- magnetic phase and reentrant transition to a glassy phase, seem to exist even when nonmagnetic zinc ions are further substituted by magnetic ions in the range below $x$ $=0.75$. To begin, we discuss the variations of the first transition with the substitution. Fig. 4 clearly shows that the onset temperature of the soft-magnetic phase $T_{\mathrm{c}}$ steeply decreases with $x$. This phenomenon can be explained by the effective weakening of $J_{\mathrm{AB}}$ due to the substitution of zinc ions in the A site, because $J_{\mathrm{AB}}$ is the driving force of the collinear ordering. In addition, we observe, at higher $x$, an expansion of the temperature range with remarkable field-dependence of $M$ in the vicinity of $T_{\mathrm{c}}$. This indicates that magnetically correlated clusters with giant magnetic moments, which can easily respond to smaller $H$, exist even at temperatures considerably higher than $T_{\mathrm{c}}$. Thus, the transition to a soft-magnetic phase is smeared by the early short-range ordering due to the substitution, although there is room for further argument as to whether such a short-range order is most correlated with "Griffiths islands" due to the stochastic inhomogeneity mentioned above, or with other types of disorders. In addition, it is crucial to clarify the variation of the critical exponents of the transition.

Next we consider the variations of the intermediate softmagnetic phase with the substitution. In the gray region of Fig. 4, we observe overall decrease of $M$. This indicates that such substitution enhances spin canting, because the saturation magnetization for robust collinear order is expected to increase with $5(1+x) \mu_{\mathrm{B}}$ in each unit formula. This enhancement of the spin canting is reasonable because the anti-ferromagnetic coupling $J_{\mathrm{BB}}$ in the $\mathrm{B}$ site becomes significant because of the weakening of $J_{\mathrm{AB}}$. Furthermore, the field-dependence of $M$ at higher $H$ becomes evident in the intermediate temperature range as $x$ increases. Because hysteresis such as the difference between $M_{\mathrm{FC}}$ and $M_{\mathrm{ZFC}}$ is undetectable, this increase of $M$ with $H$ can be attributed not to the depinning of domain walls but to the easy rotations of the canted spins. It is debatable whether the observed increase of the fragility of the canted spin state for the applied field is associated with the reduction of random anisotropy effects with the substitution.

Finally, we focus on the variation of the magnetic glassy phase with the substitution. In Fig. 4, the maximum temperature $T_{\mathrm{m}}$ of $M_{\mathrm{FC}}(T)$ increases as $x$ increases from 0.60 to 0.75 . Moreover, the decrease of $M_{\mathrm{FC}}$ below $T_{\mathrm{m}}$ becomes steep with $x$. Therefore, it can be concluded that further substitution further stabilizes the magnetic glassy phase in the wider temperature range. It is noteworthy that the extrapolated curve of the rising trend of $T_{\mathrm{m}}$ merges with that of $T_{\mathrm{c}}$ at $x=0.80$, as shown in the inset of Fig. 4. At this concentration, we observe neither the 
maximum of $M_{\mathrm{FC}}(T)$ at $T_{\mathrm{m}}$ nor the steep increase of $M$ in low $H$ at $T_{\mathrm{c}}$. In other words, both transitions vanish at $x=$ 0.80 . Therefore, it is likely that the disappearance of the intermediate soft-magnetic phase is attributed not to the arrival of $T_{\mathrm{c}}$ at absolute zero with the dilution of the Asublattice but to the further stabilization of the magnetic glassy phase at higher temperatures. Certainly, the concentration at $x=0.80$ is lower than the percolation threshold of $x_{\mathrm{c}}=0.91$ for the dilution of the A-sublattice [19]. Such stabilization of the magnetic glassy phase is not readily attributed to the random anisotropy effects because they weaken with the substitution. On the other hand, it is worthwhile to discuss the relevance of the stabilization to variation of the bond-randomness with the substitution. From the viewpoint of the molecular field acting on iron ions, the random substitution remarkably reduces its mean value in comparison with the variation of its dispersion, as discussed above. In such cases, a well-known phase diagram with reentrant spin-glass transitions has been predicted [20]. In this context, the observed behavior of the dilute spinel ferrites can be interpreted in the framework of the reentrant spin-glass picture. However, we know that the spins in the B-sublattice are topologically frustrated at $x=1.0$. Therefore, further study on the spin-glasslike behavior at $0.8<x \leq 1.0$ is required for obtaining the complete picture of this magnetic glassy phase.

\section{Concluding Remarks}

The substitution of nonmagnetic zinc ions causes the dilution of magnetic ions in the A-sublattice and consequently affects bond-randomness in the B-sublattice. In brief, the average of the random bonds varies from ferromagnetic to antiferromagnetic with the substitution. On the other hand, the random anisotropy effects due to local Jahn-Teller distortions seem to gradually weaken because the occupancy of $\mathrm{Mn}^{3+}$ ions in the B site seems to diminish with the substitution. We discuss the effects of these different types of disorder on the transition to softmagnetic phase and the reentrant transition to the glassy phase. We, however, leave room for further investigation. In order to establish the understanding of the nature of diluted ferrimagnets, a comprehensive study on their various types is necessary. Because knowledge on various types of disorders is indispensable in such studies, high throughput neutron powder diffractometers would be of further aid in the next stages of the investigation on diluted ferrimagnets.

\section{Acknowledgement}

The authors acknowledge helpful discussions with $\mathrm{T}$. Mochiku, and technical assistance from Y. Kawamura. This study was partly supported by a Grant-in-Aid for Scientific Research (\#21681014) and by the NIMS-RIKENJAEA Cooperative Research Program on Quantum Beam Science and Technology.

\section{References}

[1] J. S. Bettinger, R. V. Chopdekar, and Y. Suzuki, Appl. Phys. Lett. 94, 072505 (2009).

[2] H. Mamiya, N. Terada, T. Furubayashi, H. S. Suzuki, and H. Kitazawa, J. Magn. Magn. Mater. 322, 1561 (2010).

[3] T. Takei, J. Magn. Soc. Jpn 2, 1 (1978).

[4] S. Krupièka and P. Novák, Ferromagnetic Materials Vol. 3, Ed. E.P. Wohlfarth, North-Holland, Amsterdam (1982) Chapter 4.

[5] A. Okita, F. Saito, S. Sasaki, T. Toyoda, and H. Koinuma, Jpn. J. Appl. Phys. 37, 3441 (1998).

[6] S. Sakurai, S. Sasaki, M. Okube, H. Ohara, and T. Toyoda, Physica B 403, 3589 (2008).

[7] Jianjun Li, Hongming Yuan, Guodong Li, Yanju Liu, and Jinsong Leng, J. Magn. Magn. Mater. 322, 3396 (2010).

[8] J. L. Dormann and M. Nogues, J. Phys.: Condens. Matter 2, 1223 (1990).

[9] T. A. Anhøj, B. Bilenberg, B.n Thomsen, C. D. Damsgaard, H. K. Rasmussen, C. S. Jacobsen, J. Mygind, and S. Mørup, J. Magn. Magn. Mater. 260, 115 (2003).

[10] R. Gerber and G. Elbinger, J. Phys. C 3, 1363 (1970).

[11] T. Ishigaki, A. Hoshikawa, M. Yonemura, T. Morishima, T. Kamiyama, R. Oishi, K. Aizawa, T. Sakuma, Y. Tomota, M. Arai, M. Hayashi, K. Ebata, Y. Takano, K. Komatsuzaki, H. Asano, Y. Takano, and T. Kasao, Nuclear Instruments and Methods in Physics Research A 600, 189 (2009).

[12] R. Oishi, M. Yonemura, Y. Nishimaki, S. Torii, A. Hoshikawa, T. Ishigaki, T. Morishima, K. Mori, and T. Kamiyama, Nuclear Instruments and Methods in Physics Research A 600, 94 (2009).

[13] T. Hahn (Ed.), International Tables for Crystallography, 3rd Ed. Vol. A, Wiley, New York (1992).

[14] R. B. Griffiths, Phys. Rev. Lett. 23, 17 (1969).

[15] S. Nakashima, K. Fujita, K. Tanaka, K. Hirao, T. Yamamoto, and I. Tanaka, Phys. Rev. B 75, 174443 (2007).

[16] M. A. Hakim, M. Manjurul Haque, M. Huq, and P. Nordblad, Physica B 406, 48 (2011).

[17] H. Mamiya, M. Onoda, T. Furubayashi, J. Tang, and I. Nakatani, J. Appl. Phys. 81, 5289 (1997).

[18] S. Chikazumi, Physics of Ferromagnetism, Oxford Science Publications, New York (1997) pp. 134 160.

[19] F. Scholl and K. Binder, Z. Phys. 39, 239 (1980).

[20] M. Gabay and G. Toulouse, Phys. Rev. Lett. 47, 201 (1981). 